\title{
Benzene 2009: Health effects and mechanism of bone marrow toxicity
}

\author{
International symposium, Munich/Germany, September 7-11, 2009 \\ (Satellite meeting to EUROTOX 2009)
}

Hermann M. Bolt

(C) Springer-Verlag 2008

Benzene, a major commodity chemical, has been known to cause bone marrow damage since the turn of the 20th century. Chronic exposure to benzene results in decreases in the numbers of red blood cells, white blood cells, and/or platelets in circulating blood and can result in aplastic anemia, myelodysplastic syndrome, acute myelogenous leukemia and perhaps other forms of leukemia. Furthermore, observations on benzene-induced leukemia command a broader audience because of its marked similarity with leukemias that appear in people who have undergone successful chemotherapy with a variety of cancer chemotherapeutic agents or radiation and then develop acute myelogenous leukemia several years later. This phenomenon has been referred to as "second cancer."

The expansion of our understanding of bone marrow function and pathophysiology since the 1950's has permitted extensive exploration of mechanisms of leukemogenesis. Thus, advances in the technologies of modern molecular biology and genomic studies have led to recent mechanistic findings which have increased our understanding of the etiologies of benzene-induced bone marrow damage and the factors that modify individual susceptibility to benzene-induced diseases. The program of the symposium include an overview of current concepts of bone marrow organization and function; mechanisms of therapy-induced acute myelogenous leukemia ( $t$-AML); recent studies on the epidemiology of benzene-induced bone marrow pathology; new aspects of exposure assessment; exploration of the range of diseases caused by benzene in humans; mechanisms of benzene-induced bone marrow damage; the application of new testing technologies (transgenic models, genomics) to understanding benzene toxicity; and organization of available data and data gaps by the WHO IPCS mode of action (MOA)/human relevance framework to inform the process of risk assessment.

The symposium will comprise plenary and platform presentations, a panel discussion and poster sessions. Peerreviewed proceedings of the symposium will be published. Abstracts for volunteer presentations are invited and may also be published in the proceedings. Most will be presented as posters, but after review by the organizers some will be selected for platform presentations. For further information go to:

http://www.tum-benzenesymposium.de

or contact:

rsnyder@eohsi.rutgers.edu

or

helmut.greim@1rz.tu-muenchen.de.
H. M. Bolt ( $\square)$

Leibniz Research Centre for Working,

Environment and Human Factors,

Institut für Arbeitsphysiologie,

an der Universität Dortmund, Ardeystr. 67,

44139 Dortmund, Germany

e-mail: bolt@ifado.de 